\title{
Validación del modelo de cinco factores de la Escala de Actitud hacia la Estadística en estudiantes mexicanos de psicología
}

\author{
Validation of the five-factor model of the Attitude Scale towards Statistics
}

in mexican psychology students

José Moral de la Rubia ${ }^{1}$, Mónica Teresa González Ramírez², René Landero

Hernández $^{3}$ y Lucía del Carmen Quezada Berumen ${ }^{4}$

\begin{abstract}
${ }^{1}$ Doctor en Filosofía y Ciencias de la Educación, Sección de Psicología. Profesor-investigador de la Facultad de Psicología de la Universidad Autónoma de Nuevo León, México. E-mail: jose moral@hotmail.com, jose.morald@uanl.edu.mx

${ }^{2}$ Doctora en Psicología. Profesora-investigadora de la Facultad de Psicología de la Universidad

Autónoma de Nuevo León, México. E-mail: monica.gonzalezrz@uanl.edu.mx

${ }^{3}$ Doctor en Filosofía con Especialidad en Trabajo Social y Políticas Comparadas de Bienestar Social. Profesor-investigador de la Facultad de Psicología de la Universidad Autónoma de Nuevo León, México. E-mail: rene.landerohr@uanl.edu.mx

${ }^{4}$ Doctora en Filosofía con Orientación en Psicología. Profesora-investigadora de la Facultad de Psicología de la Universidad Autónoma de Nuevo León, México.

E-mail: luciaqb86@msn.com, lucia.quezadabrmn@uanl.edu.mx

Facultad de Psicología, Universidad Autónoma de Nuevo León. Monterrey, Nuevo León, México.
\end{abstract}

\section{Resumen}

La actitud positiva hacia la estadística promueve el estudio y uso de esta disciplina fundamental para analizar datos; de ahí la relevancia de evaluar el nivel actitudinal y desarrollar estrategias que lo potencien. La Escala de Actitud hacia la Estadística (EAE-25) de Auzmendi es uno de los instrumentos más usados en español para medir esta actitud, pero no se ha validado en México, lo que es importante para su uso en este país y para aportar evidencias de validez. Los objetivos del estudio fueron contrastar el modelo propuesto originariamente, comprobar su consistencia interna, describir su distribución, comparar los promedios entre sexos y verificar su validez concurrente. Este estudio instrumental usó un muestreo no probabilístico. Se analizó una muestra de 383 estudiantes de psicología mexi- canos (73.6 \% mujeres y $26.4 \%$ hombres) que respondieron a dos escalas de actitud. El modelo de cinco factores (confianza, ansiedad, agrado, utilidad y motivación) mostró buen ajuste a los datos. Sus factores presentaron confiabilidad y validez discriminante. La distribución de las puntuaciones en EAE-25 fue normal. La actitud se polarizó, aproximadamente, en cuatro de cada diez estudiantes hacia la aceptación y en uno de cada diez hacia el rechazo. No hubo diferencias de medias entre sexos. La EAE-25 tuvo correlación alta con actitud hacia la investigación y baja con la calificación en la materia de investigación del semestre anterior. Se concluye que la EAE-25 presenta consistencia interna y validez de constructo.

Palabras clave: estudio de validación, actitud hacia la estadística, actitud hacia la ciencia, rendimiento académico, análisis factorial confirmatorio 


\section{Abstract}

The positive attitude towards statistics promotes the study and use of this fundamental discipline to analyze data, hence the relevance of evaluating the attitudinal level and developing strategies that promote a positive attitude. The Auzmendi's Scale of Attitude towards Statistics (EAE-25) is one of the most used instruments in Spanish language to assess this attitude; however, it has not been validated in Mexico, which is important for its use in this country and to provide evidence of validity. The objectives of the study were to test the originally proposed model, to verify its reliability, to describe its distribution, to compare the averages between both sexes, and to verify its concurrent validity.

A non-probabilistic sampling was used in this instrumental study. A sample of 383 Mexican psychology students was analyzed (73.6\% women and $26.4 \%$ men). The EAE-25 and a scale assessing attitude towards research were applied. Confirmatory factor analysis was used to test the Auzmendi's fivefactor model. The parameter estimation was performed by unweighted least squares, using polychoric correlation matrix. The internal consistency was estimated by the McDonald's omega coefficient $(\omega)$, the convergent validity by the average variance extracted (AVE), and the discriminant validity by the heterotrait-monotrait ratio of correlations (HTMT). The mean differences between both sexes were tested through the t-test and concurrent validity through Pearson's correlation coefficients.

The model of five correlated factors (confidence, anxiety, pleasure, usefulness, and motivation) showed good fit $\left(\chi^{2} / d f=1.79\right.$, $G F I=.98, A G F I=.97, N F I=.97, C F I=.99$, RMSEA $=.04$, and SRMR $=.07)$. The five factors showed reliability $(\omega>.80$ in confidence, anxiety, pleasure, and usefulness, and $\omega=.72$ in motivation) and discriminant validity $(H T M T<.85$ in nine out of ten factor pairs, and $<.90$ in the pair of the anxiety and confidence factors). The values of AVE were higher than .50 in the factors of confidence, anxiety and pleasure, which shows convergent validity in these three factors. A value of AVE very close to .50 in utility $(\mathrm{AVE}=.49)$ was compensated by an omega coefficient of .82, which suggests an acceptable convergent validity. However, the value of AVE was lower than .50 in motivation $(\mathrm{AVE}=.34)$.

The EAE-25 and its factors presented normal distribution, with the exception of the motivation factor. The scores in the scale and its factors were obtained through the quotient between the sum of the items scored in the sense of acceptance attitude and the number of items added. These scores were interpreted in an absolute sense, by dividing the continuum from 1 to 5 into five intervals in correspondence with the five ordered categories of response to the items: $[1,1.8)$ y $[1.8$, $2.6)$ rejection, $[2.6,3.4)$ neutral, and $[3.4,4.2)$ $\mathrm{y}[4.2,5]$ acceptance. The average attitude was positive in confidence $(M=3.53)$ and utility $(M=3.52)$, and neutral in the EAE-25 total score and its other three factors. The EAE-25 total scores were polarized towards acceptance in approximately four out of ten students and towards the rejected in approximately one out of ten; in the rest, they were neutral. There were no differences of means between both sexes.

The EAE-25 total score had a high correlation with the total score in the scale of attitude towards research $(r=.52, p<.001)$ and low with the qualification in the research subject of the previous semester $(r=.13, p<.001)$.

It is concluded that EAE-25 presents internal consistency and construct validity. It is suggested to use this scale in applied research in the field of university education and upper secondary education.

Keywords: validation study, attitude toward statistics, attitude toward science, academic performance, confirmatory factor analysis 


\section{Introducción}

La estadística es una herramienta fundamental para analizar datos muestrales. A su vez, la investigación con datos procedentes de muestras es necesaria para el desarrollo de una ciencia empírica. De ahí que la estadística forme parte del currículum académico en la mayoría de las carreras científicas, incluida la psicología. En esta carrera, la enseñanza de conceptos estadísticos presenta problemas didácticos específicos debido a que los estudiantes acceden con bases matemáticas muy heterogéneas (Comas, Martins, Nascimento y Estrada, 2017).

La actitud es la tendencia del individuo a evaluar de forma favorable o desfavorable un objeto, ya sea concreto o abstracto (Bautista, Morales, Dórame y Peralta, 2016). Al estudiante de psicología se le presenta el objeto de la estadística, ante el cual se forman actitudes integradas por reflexiones, sentimientos y experiencias acumulados a lo largo del tiempo de aprendizaje tanto de la estadística como de las matemáticas y la metodología de la investigación (Bautista et al., 2016). Estas actitudes pueden variar de negativas, con un claro rechazo hacia la estadística, a positivas, con una abierta aceptación. No obstante, la actitud positiva hacia la estadística es la que promueve su estudio y uso, con el beneficio consecuente para la disciplina científica. Por el contrario, las actitudes negativas y las carencias en conocimientos estadísticos pueden motivar que la investigación se limite a planteamientos rudimentarios cuando se requerirían análisis más finos. De ahí la relevancia de evaluar el nivel actitudinal y desarrollar estrategias que potencien una actitud positiva.

Rodríguez Feijóo (2011) encontró una actitud neutra hacia la estadística en los estudiantes argentinos que cursaban carreras humanísticas, en comparación con una actitud favorable en los que cursaban carreras orientadas hacia la matemática. Un resultado afín de actitud neutra fue reportado por Ruiz de Miguel (2015) en estudiantes españoles de ciencias de la educación. Por otra parte, en estudiantes estadounidenses de psicología, Walker y Brakke (2017) encontraron que las actitudes eran moderadamente positivas, al igual que Comas et al. (2017) en estudiantes españoles y Bautista et al. (2016) en estudiantes mexicanos de esta misma carrera.

Por cuestiones culturales de género, pudiera pensarse que la actitud hacia la estadística es más positiva en hombres que en mujeres (Bautista et al., 2016). Gil (1999), al revisar las diversas investigaciones, encontró hallazgos contradictorios y, en su estudio con estudiantes españoles de pedagogía, el sexo resultó un factor no significativo. Estrada, Batanero y Fortuny (2004) tampoco hallaron que el sexo fuera un factor diferencial en la actitud de normalistas españoles en formación. A su vez, Pérez, Aparicio, Bazán y Abdounur (2015) tampoco hallaron diferencias entre mujeres y hombres en estudiantes universitarios colombianos. En México, Salinas y Mayén (2016) reportaron promedios estadísticamente equivalentes en la actitud hacia la estadística entre ambos sexos en estudiantes mexicanos de bachillerato. Bautista et al. (2016) reportaron promedios más altos en los factores de percepción de la utilidad de la estadística y ansiedad en mujeres que en hombres, así como un promedio más alto en confianza ante la estadística en hombres que en mujeres, aunque no hubo diferencias entre ambos sexos en la puntuación total de la EAE-25 y sus otros dos factores.

Uno de los instrumentos más usados en español para medir esta actitud es la Escala de Actitud hacia la Estadística de 25 ítems (EAE-25; Auzmendi, 1992) que está integrada por cinco factores: utilidad, ansiedad, confianza, agrado y motivación (Auzmendi, 1992). En estudiantes de economía españoles, Rojas, Escalera, Moreno y García (2017) validaron el modelo de cinco factores, obteniendo consistencia interna aceptable en los factores (de $\alpha=.72$ a .79) y buena en la escala $(\alpha=$ $.80)$. No obstante, la EAE-25 no se ha validado en México, lo que es importante no solo 
para su aplicación en este país, sino también para aportar evidencias de validez en torno al modelo de cinco factores, el cual ha sido cuestionado frente a soluciones de cuatro (Méndez y Macía, 2007) y tres factores (Tejero y Castro, 2011).

Considerando estos huecos en la investigación, el propósito del presente estudio es validar la EAE-25 en una muestra de estudiantes de psicología mexicanos. Para este fin, se plantearon cinco objetivos: 1) contrastar el modelo factorial propuesto originariamente por Auzmendi (1992), 2) verificar la consistencia interna y validez convergente y discriminante de cada variable latente dentro del modelo de cinco factores correlacionados, 3) describir la distribución de la escala y sus factores, 4) comparar los promedios entre los factores y entre mujeres y hombres y 5) comprobar su validez de constructo concurrente en relación con la calificación en la materia de investigación del semestre anterior y la actitud hacia la investigación.

\section{Método}

\section{Diseño}

Este estudio empírico fue de tipo instrumental. Su diseño fue ex post facto de corte transversal. Se usó un muestreo no probabilístico con la aplicación individual de un cuestionario en línea.

\section{Participantes}

Los criterios de inclusión fueron ser estudiante de la licenciatura en psicología de la universidad pública del estado de Nuevo León en México en cualquiera de sus 10 semestres $\mathrm{y}$ dar el consentimiento informado. Se incluyeron los 10 semestres, ya que cada semestre tiene una materia del eje metodológico que está centrada (segundo y cuarto semestre) o incluye contenidos de análisis de datos y estadística. El criterio de eliminación fue dejar preguntas $\sin$ responder en el cuestionario en línea presentado a través de la plataforma Survey Monkey. Se eliminaron 29 casos por datos incompletos. La muestra analizada fue de 383 estudiantes con todos sus datos completos.

El $73.6 \%$ de los participantes fueron mujeres y el $26.4 \%$, hombres, en correspondencia con los porcentajes por sexo en la población de estudiantes de psicología, de la cual fue extraída la muestra $\left(\chi^{2}[1, N=383]=\right.$ $3.84, p=.536)$. La muestra quedó integrada por estudiantes de los 10 semestres de la carrera, aunque predominaron estudiantes de los cuatro primeros semestres que representan el 84.6 $\%$ de la función empírica de distribución. La media aritmética de edad fue de 19.40 años, con una desviación estándar de 2.96. La media aritmética de las calificaciones en la materia de investigación del semestre anterior (con un rango potencial de 0 a 100) fue $88.17 \mathrm{y} \mathrm{su}$ desviación estándar, 8.07. Una calificación es aprobatoria si es de al menos 70 puntos. La distribución de estas calificaciones con forma acampanada mostró mesocurtosis $\left(Z_{K}=-.45\right)$, pero ligera asimetría negativa $\left(Z_{S k}=-3.68\right)$, por lo que no se ajustó a la normalidad (prueba de D'Agostino y Pearson: $K^{2}=13.75, p=.001$ ). Cabe aclarar que la materia de investigación del semestre anterior en los estudiantes de primer semestre se refiere a la asignatura de metodología de la investigación científica cursada en el último año de preparatoria.

\section{Instrumentos de medida}

El cuestionario en línea estaba integrado por la solicitud de consentimiento informado, dos preguntas sobre información sociodemográfica (sexo y edad), cinco preguntas sobre información académica, la EAE-25 y una escala de actitudes hacia la investigación.

La EAE-25 tiene 25 ítems con rango de respuesta tipo Likert de cinco puntos (desde 1: totalmente en desacuerdo hasta 5: totalmente de acuerdo). Los 10 ítems negativos a recodificar fueron los ítems 2, 5, 7, 10, 12, 15, 16, 17,22 y 25 . A mayor puntuación en la escala y 
los factores, más favorable es la actitud hacia la estadística. En el estudio de Auzmendi (1992) se encontró una consistencia interna de .87 a .91 . Definido el número de factores por el criterio de autovalores de la matriz de correlaciones mayores que uno y extraídos por el método de Componentes Principales con rotación Varimax, se obtuvieron cinco factores con cinco ítems cada uno: utilidad (ítems 1, 6, 11, 20 y 21) o valor que el estudiante otorga a la estadística para su futura vida profesional, ansiedad (ítems 2, 7, 12, 17 y 22) o temor que el estudiante manifiesta ante la asignatura, confianza (ítems 3, 8, 13, 18, 23) o sentimiento de seguridad que provoca su habilidad en estadística, agrado (ítems 4, 9, 14,19 y 24) o disfrute que provoca el trabajo con la estadística y motivación (ítems 5, 10, 15,16 y 25) o inclinación hacia el estudio y utilización de la estadística. Sus valores de consistencia interna $\alpha$ variaron entre .61 y .84 (Auzmendi, 1992).

Se utilizó la adaptación de la Escala de Actitudes hacia la Investigación hecha en población mexicana (EAI-32; Quezada, Moral y Landero, 2019). A la escala original de 34 ítems de Aldana, Caraballo y Babativa (2016), Quezada et al. (2019) le añadieron un ítem sobre un aspecto conductual de la actitud (ítem 35: "para ser sincero/a realmente lo que menos hago es leer") y eliminaron tres ítems por baja consistencia interna ( 28,30 y 32), por lo que se redujo a 32 ítems. En su estudio realizado con una muestra de 392 estudiantes de psicología mexicanos, el número de factores por el análisis paralelo de Horn (1965) y la media mínima de las correlaciones parciales al cuadrado (Velicer, 1976) fue de tres. Al extraer los factores desde la matriz de correlación policórica por mínimos cuadrados no ponderados se explicó el 45.3 $\%$ de la varianza de los ítems. Tras la rotación oblicua de la matriz de cargas factoriales por el método Promax, se configuró un primer factor con trece ítems directos sobre aspectos afectivos y conductuales (ítems 2, 3, 6, 8, 10, $11,13,16,17,18,21,24$ y 25; $\alpha$ ordinal: .90).
El segundo factor quedó formado por nueve ítems sobre aspectos cognitivos (ítems 7, 12, 15, 20, 22, 26, 29, 31 y 33; $\alpha$ ordinal: .89). El tercer factor quedó integrado por los diez ítems inversos sobre aspectos conductuales y afectivos (ítems 1, 4, 5, 9, 14, 19, 23, 27, 34 y $35 ; \alpha$ ordinal: .87). En el análisis factorial confirmatorio, estos tres factores mostraron validez convergente y discriminante. El ajuste a los datos, estimados los parámetros por mínimos cuadrados no ponderados desde la matriz de correlación policórica, fue bueno por siete índices $\left(\chi^{2} / \mathrm{gl}=1.23<2\right.$; GFI $=.97$, $\mathrm{CFI}=.99$, NFI $=.95$ y RFI $=.95 \geq .95$; AGFI $=.96>.90$ y RMSEA $=.02<.05)$ y aceptable por uno $($ SRMR $=.07<.10)$.

En la presente muestra, la consistencia interna de los 32 ítems fue excelente (ordinal $\alpha$ : 94) y las puntuaciones en EAI-32 siguieron una distribución normal (prueba de D'Agostino y Pearson: $K^{2}=5.02, p=.081$ ). La distribución del factor de ítems directos de aspectos afectivos-conductuales no se ajustó a la normalidad $\left(K^{2}=9.12, p=.010\right)$, al presentar ligera leptocurtosis $\left(Z_{K 3}=2.73>1.96\right)$; su consistencia interna fue excelente ( $\alpha$ ordinal: .90$)$. La distribución del factor de aspectos cognitivos, con asimetría negativa $\left(Z_{S k}=-4.87\right) \mathrm{y}$ leptocurtosis $\left(Z_{K 3}=5.28>1.96\right)$, no se ajustó a la normalidad $\left(K^{2}=51.63, p<.001\right)$; su consistencia interna fue buena ( $\alpha$ ordinal: .89 ). La distribución del factor de ítems inversos de aspectos afectivos y conductuales se ajustó a la normalidad $\left(K^{2}=.25, p=.884\right)$ con consistencia interna buena ( $\alpha$ ordinal: .86).

\section{Procedimiento}

En los salones de clase, maestros del área de investigación invitaron a sus alumnos a que contestaran un cuestionario en línea. Además, se solicitó a los estudiantes que difundieran el enlace con compañeros a través de sus redes sociales. El enlace estuvo disponible de octubre a diciembre de 2017.

Entre las instrucciones se pidió a los participantes responder a todas las preguntas. La 
participación en el estudio fue voluntaria y no remunerada. Se garantizó la confidencialidad en el tratamiento de los datos. A tal fin, no se solicitó ningún dato de identificación personal. Además, había una dirección de correo para resolver cualquier duda y los nombres de los responsables de la investigación estaban disponibles. Así, se siguieron las normas de investigación de la American Psychological Association (2017).

\section{Análisis de datos}

Para el primer objetivo de comprobar el modelo de Auzmendi (1992), se realizó análisis factorial confirmatorio. Se optimizó la función de discrepancia por mínimos cuadrados no ponderados, usando como datos de entrada la matriz de momentos (media aritmética, desviación estándar y correlación policórica). El error estándar de las estimaciones y el contraste de la significación se realizó por percentiles corregidos de sesgo con la extracción de 2,000 muestras aleatorias. El ajuste de los modelos se valoró por medio de ocho índices: $\chi^{2} / g l$ : chi-cuadrada relativa, GFI: índice de bondad de ajuste, $A G F I$ : índice de bondad de ajuste corregido, $N F I$ : índice normado de ajuste, $C F I$ : índice comparativo de ajuste, $R F I$ : índice relativo de ajuste, $S R M R$ : residuo estandarizado cuadrático medio y RMSEA: error de aproximación cuadrático medio. Se estipuló que valores de $\chi^{2} / g l$ menores o iguales a 2, GFI, NFI, CFI y $R F I$ mayores o iguales a $.95, A G F I$ mayores o iguales a .90 , y $S R M R$ y $R M S E A$ menores o iguales a .05 reflejaban un buen ajuste. Del mismo modo, valores de $\chi^{2} / g l$ menores o iguales a 3, GFI, NFI, CFI y RFI mayores o iguales a .90, $A G F I$ mayores o iguales a .85 , $S R M R$ menores a .08 y RMSEA menores a.10, indicaban un ajuste aceptable (Byrne, 2016).

Para el segundo objetivo, la consistencia interna de cada factor se determinó por el coeficiente omega de McDonald $(\omega)$. Se estipuló que valores de $\omega$ entre .70 y .79 reflejan una consistencia interna aceptable, entre .80 y .89 , buena y mayores o iguales a .90 , excelente (Viladrich, Angulo y Doval, 2017). La validez convergente de cada factor se evaluó por la varianza media extraída (Average Variance Extracted, AVE, por sus siglas en inglés) y se consideró que un valor de AVE mayor a .50 muestra validez convergente (Fornell y Larcker, 1981). La validez discriminante entre factores se estableció a través de la proporción hetero rasgo/mono rasgo de las correlaciones (HTMT) o cociente entre la media aritmética de las $n^{*} m$ correlaciones no redundantes de los ítems cruzados entre dos factores y la media geométrica de las medias de las $[n *(n-1)] / 2 \circ[m *(m-1)] / 2$ correlaciones no redundantes entre los ítems de cada factor. Se estipuló que un valor de HTMT menor o igual a .85 refleja validez discriminante o al menos menor o igual a .90 (Henseler, Ringle y Sarstedt, 2015).

Para el tercer objetivo de describir las distribuciones, el ajuste a la normalidad se contrastó por la prueba de D'Agostino y Pearson $\left(K^{2}\right)$, usando las fórmulas corregidas de sesgo de Fisher (Urzúa, 1996).

Para el cuarto objetivo, la comparación de medias entre los factores se hizo por análisis de varianza de medidas repetidas y las comparaciones a posteriori por la prueba de Sidak (1967). La comparación de medias en EAE-25 y sus factores entre ambos sexos se hizo por la prueba $t$ de Student para dos muestras independientes. Se cumplió el supuesto de normalidad, salvo en el factor de motivación; su incumplimiento fue por leptocurtosis, ante lo cual estas pruebas son robustas (Thabane et al., 2013).

Para el quinto objetivo de validez concurrente, las correlaciones se calcularon por el coeficiente de Pearson ( $r$ y y su significación $\left(\mathrm{H}_{0}: r=0\right)$ se contrastó por la prueba $t$ con n - 2 grados de libertad. Se comprobó la normalidad bivariada por las pruebas basadas en la asimetría ( $U$-test) y curtosis ( $W$-test). Un valor de probabilidad mayor que .05 muestra un ajuste a la normalidad bivariada (Tyler, Critchley, Dümbgen y Oja, 2009). En caso de 
incumplimiento de este supuesto, la significación de las correlaciones se comprobó por su estimación por intervalo con un nivel de confianza al $95 \%$ a través de muestreo repetitivo con la simulación de 2.000 muestras por el método de percentiles (Bishara y Hittner, 2015). Valores de $|r|$ menores a .10 se interpretaron como una fuerza de asociación trivial, entre .10 y .29 débil, entre .30 y .49 moderada, entre .50 y .69 fuerte, entre .70 y .89 muy fuerte, y mayor o igual a .90 perfecta (Byrne, 2016).

El nivel de significación se fijó en .05 y los cálculos se hicieron con los programas SPSS 24, módulo R versión 2.4 para SPSS 24, AMOS 16 y Excel 2013.

\section{Resultados}

\section{Contraste del modelo de cinco factores correlacionados}

La solución del modelo de cinco factores correlacionados fue admisible.

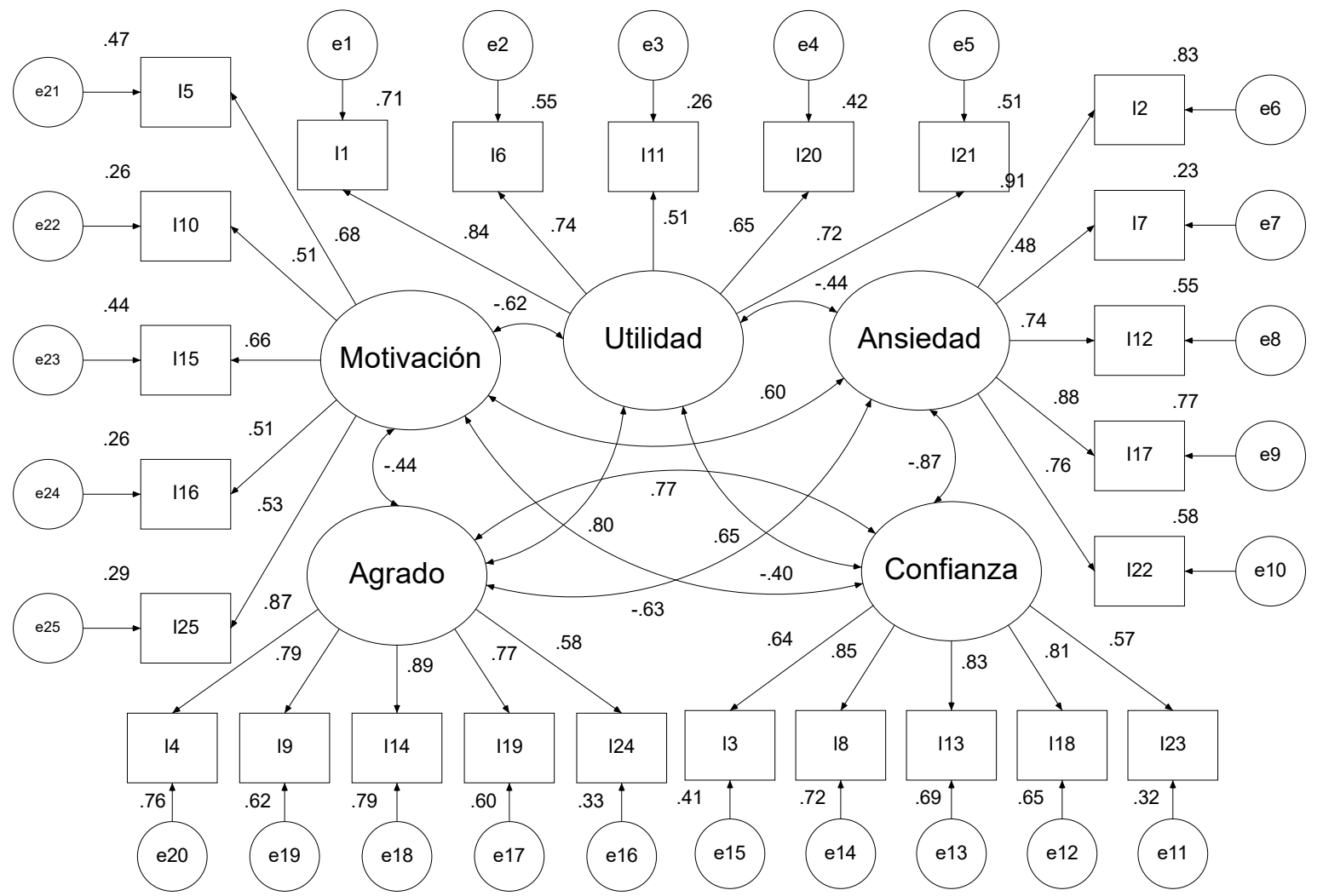

Figura 1. Modelo de cinco factores correlacionados estimado por mínimos cuadrados no ponderados.

Todos los parámetros fueron significativos. Los factores ansiedad (AVE $=.59$ y $\omega=.87$ ), confianza (AVE $=.56$ y $\omega=.86)$ y agrado $(\mathrm{AVE}=.62$ y $\omega=.89)$ mostraron validez convergente $(\mathrm{AVE}>.50)$ y valores de consistencia interna buenos $(.80 \leq \omega<.90)$. El factor de utilidad tuvo una AVE de .49 y consistencia interna buena $(\omega=.82)$. No obstante, el factor de motivación no mostró validez convergente $(\mathrm{AVE}=.34<.50)$ y su consistencia interna fue aceptable $(.70 \leq \omega=.72<.80)$. 
Tabla 1

Estadísticos descriptivos y contraste de la normalidad $(N=383)$.

\begin{tabular}{|c|c|c|c|c|c|c|}
\hline Estadísticos & $\begin{array}{c}\text { EAE-25 } \\
\text { P. total }\end{array}$ & Utilidad & Ansiedad & Confianza & Agrado & Motivación \\
\hline Min & 1.68 & 1 & 1 & 1 & 1 & 1 \\
\hline Máx & 4.92 & 5 & 5 & 5 & 5 & 5 \\
\hline$M$ & 3.25 & 3.52 & 3.26 & 3.53 & 2.76 & 3.17 \\
\hline Mo & 3.08 & 3.40 & 3 & 3.40 & 3 & 3 \\
\hline$D E$ & .56 & .70 & .79 & .71 & .78 & .63 \\
\hline$Z_{S k}$ & 1.98 & .02 & -.66 & -1.52 & 1.56 & 1.58 \\
\hline$Z_{K 3}$ & 1.25 & 1.06 & .90 & 1.22 & .50 & 3.12 \\
\hline P10 & 2.58 & 2.68 & 2.20 & 2.60 & 1.80 & 2.40 \\
\hline$P 20$ & 2.84 & 3 & 2.60 & 3 & 2 & 2.76 \\
\hline$P 25$ & 2.92 & 3 & 2.80 & 3 & 2.20 & 2.80 \\
\hline P30 & 3 & 3.20 & 3 & 3.20 & 2.40 & 2.80 \\
\hline P40 & 3.08 & 3.40 & 3 & 3.40 & 2.60 & 3 \\
\hline$P 50$ & 3.20 & 3.40 & 3.20 & 3.60 & 2.80 & 3.20 \\
\hline$P 60$ & 3.32 & 3.60 & 3.40 & 3.60 & 3 & 3.20 \\
\hline$P 70$ & 3.48 & 3.80 & 3.60 & 3.96 & 3 & 3.40 \\
\hline P75 & 3.56 & 3.80 & 3.80 & 4 & 3 & 3.60 \\
\hline$P 80$ & 3.69 & 4.20 & 3.80 & 4.20 & 3.24 & 3.60 \\
\hline$P 90$ & 4 & 4.40 & 4.20 & 4.40 & 3.80 & 4 \\
\hline$K^{2}$ & 5.46 & 1.13 & 1.25 & 3.80 & 2.69 & 12.25 \\
\hline$p$ & .065 & .570 & .535 & .149 & .261 & .002 \\
\hline
\end{tabular}

Notas: Utilidad $=(\mathrm{i} 1+\mathrm{i} 6+\mathrm{i} 11+\mathrm{i} 20+\mathrm{i} 21) / 5$, Ansiedad $=[(6-\mathrm{i} 2)+(6-\mathrm{i} 7)+(6-\mathrm{i} 12)+(6-\mathrm{i} 17)+(6-\mathrm{i} 22)] / 5$, Confianza $=$ $(\mathrm{i} 3+\mathrm{i} 8+\mathrm{i} 13+\mathrm{i} 18+\mathrm{i} 23) / 5$, Agrado $=(\mathrm{i} 4+\mathrm{i} 9+\mathrm{i} 14+\mathrm{i} 19+\mathrm{i} 24) / 5$ y Motivación $=[(6-\mathrm{i} 5)+(6-\mathrm{i} 10)+(6-\mathrm{i} 15)+(6-\mathrm{i} 16)$ $+(6-i 25)] / 5$. Estadísticos: $Z_{S k}$ : valor estandarizado del coeficiente de asimetría basado en el tercer momento, $Z_{K 3}:$ valor estandarizado del exceso de curtosis, $P 10$ a $P 90$ : percentiles. Contraste de la normalidad por la prueba de D'Agostino y Pearson: $K^{2}=$ estadístico de contraste, $p=$ probabilidad en un contraste a dos colas.

Las diez correlaciones entre los factores fueron significativas y la varianza compartida osciló de $16 \%$ a $64 \%$ con media de $40 \%$. Solo la proporción hetero rasgo/mono rasgo entre ansiedad y confianza fue mayor que .85, aunque menor que $.90(H T M T=.86)$. En los nueve casos restantes, los valores de HTMT variaron de .40 a .80 con una media de .59 . Por tanto, hubo validez discriminante entre los factores. El ajuste del modelo fue bueno por siete índices $\left(\chi^{2} / g l=1.79<2 ; G F I=.98\right.$, $N F I=.97, C F I=.99$ y $R F I=.96>.95 ; A G F I$ $=.97>.90$ y $R M S E A=.04<.05)$ y aceptable por uno $(S R M R=.07<.10)$. 


\section{Distribuciones de las puntuaciones en EAE-25 y sus cinco factores}

Las puntuaciones en la escala y los cinco factores se obtuvieron a través del cociente entre la suma de los ítems puntuados en sentido de actitud de aceptación y el número de ítems sumados. Se interpretaron en sentido absoluto, al dividir el continuo de puntuaciones de 1 a 5 en cinco intervalos en correspondencia con las cinco categorías ordenadas de respuesta a los ítems: $[1 ; 1.8$ ): 1 (muy en desacuerdo), $[1.8 ; 2.6)$ : 2 (en desacuerdo), [2.6; 3.4$): 3$ ni de acuerdo ni en desacuerdo), [3.4; 4.2): 4 (de acuerdo) y [4.2; 5): 5 (muy de acuerdo).

La tendencia central (media aritmética, mediana y moda) de los factores de utilidad y confianza reflejó una actitud de aceptación ( $\geq 3.4$ ). No obstante, los valores de tendencia central de la escala y los factores de falta de ansiedad, agrado y motivación quedaron en el intervalo [2.6; 3.4), que corresponde a una actitud neutra (Tabla 1).

En el $10 \%$ de los estudiantes, IC $95 \%$ ( $7 \%, 13 \%)$, sus puntuaciones en EAE-25 correspondieron a una actitud de rechazo, en el $53 \%$, IC $95 \%$ (48\%, $58 \%)$, neutra, y en el $37 \%$, IC $95 \%$ (32\%, $42 \%)$, de aceptación. La diferencia entre estos tres porcentajes fue significativa, $\chi^{2}[2, N=383]=108.10, p<.001$, dominando la actitud neutra. En el estudio de Bautista et al. (2016) con la EAE-25 en estudiantes universitarios mexicanos, estos porcentajes en la división de ciencias sociales, fueron $15 \%$ (rechazo), $40 \%$ (neutralidad) y $45 \%$ (aceptación). Al compararlos con los presentes porcentajes, la diferencia fue signi- ficativa, $\chi^{2}[2, N=383]=27.13, p<.001$.

Las puntuaciones en utilidad, falta de ansiedad, confianza y agrado mostraron perfiles acampanados, simétricos y mesocúrticos en los histogramas, por lo que se ajustaron a la normalidad. En el perfil de la puntuación total del EAE-25 acampanado y simétrico, hubo muy ligera asimetría positiva $\left(Z_{K 3}=1.98\right)$, pero se pudo mantener la hipótesis nula de normalidad. El factor de motivación presentó ligera leptocurtosis, lo que provocó desviación de la normalidad (Tabla $1)$.

\section{Comparaciones de medias entre los factores y ambos sexos}

Al comparar las medias entre los cinco factores, hubo diferencia estadísticamente significativa, $F(3.18,1215.69)=141.17, p<$ .001 . No se asumió equivalencia de varianzas en las puntuaciones diferenciales entre los 10 factores por la prueba de la esfericidad de Mauchly (1940), $\chi^{2}[9, N=383]=239.99$, $p<.001$, por lo que se usó la corrección de Greenhouse y Geisser (1959) para los grados de libertad, $\varepsilon=.80$. El tamaño del efecto fue grande $\left(\eta_{\mathrm{p}}{ }^{2}=.27\right)$. Al hacer las comparaciones entre pares de factores por la prueba de Sidak (1967), ocho de las diez diferencias fueron significativas. Las medias de utilidad y confianza, $D M=-.02$, IC 95\% (-.12; .08), $p=$ $1, \mathrm{y}$ las de motivación y falta de ansiedad: $D M$ $=-.09$, IC 95\% (-.02; .20), $p=.184$, fueron estadísticamente equivalentes. La media en falta de ansiedad fue significativamente más baja que las otras cuatro medias (Tabla $1 \mathrm{y}$ Figura 2). 


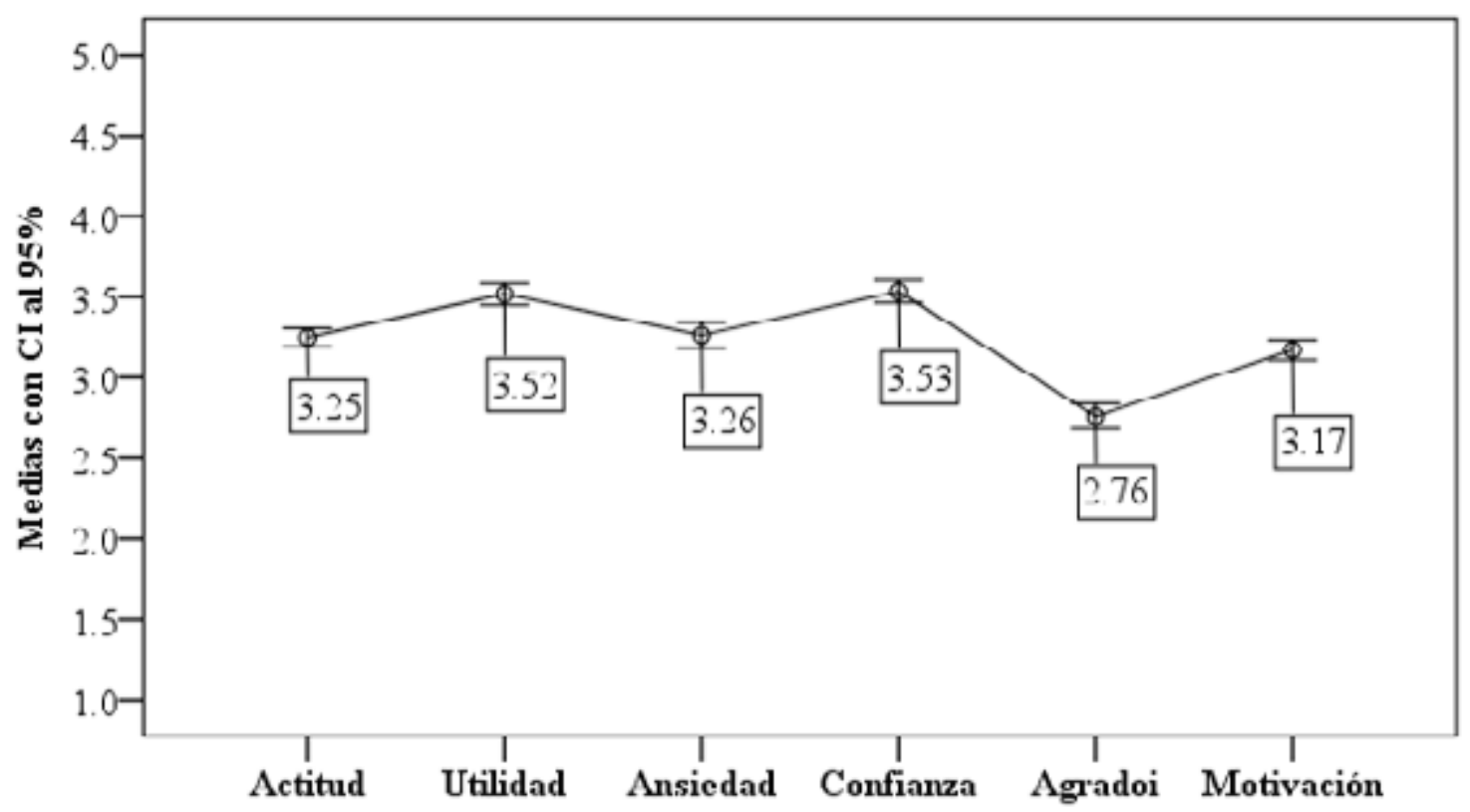

Figura 2. Diagrama de medias de la EAE-25 y sus cinco factores en la muestra conjunta.

Al comparar las medias entre sexos por la prueba $t$ de Student, la diferencia no fue significativa ni en el factor general ni en los cinco factores, pudiéndose asumir igualdad de varianzas en todos los casos por la prueba de Levene. Cabe señalar que el promedio en la calificación en la materia de investigación en el semestre previo fue estadísticamente equivalente entre ambos sexos, $t[381]$ $=-.39, p=.695$.

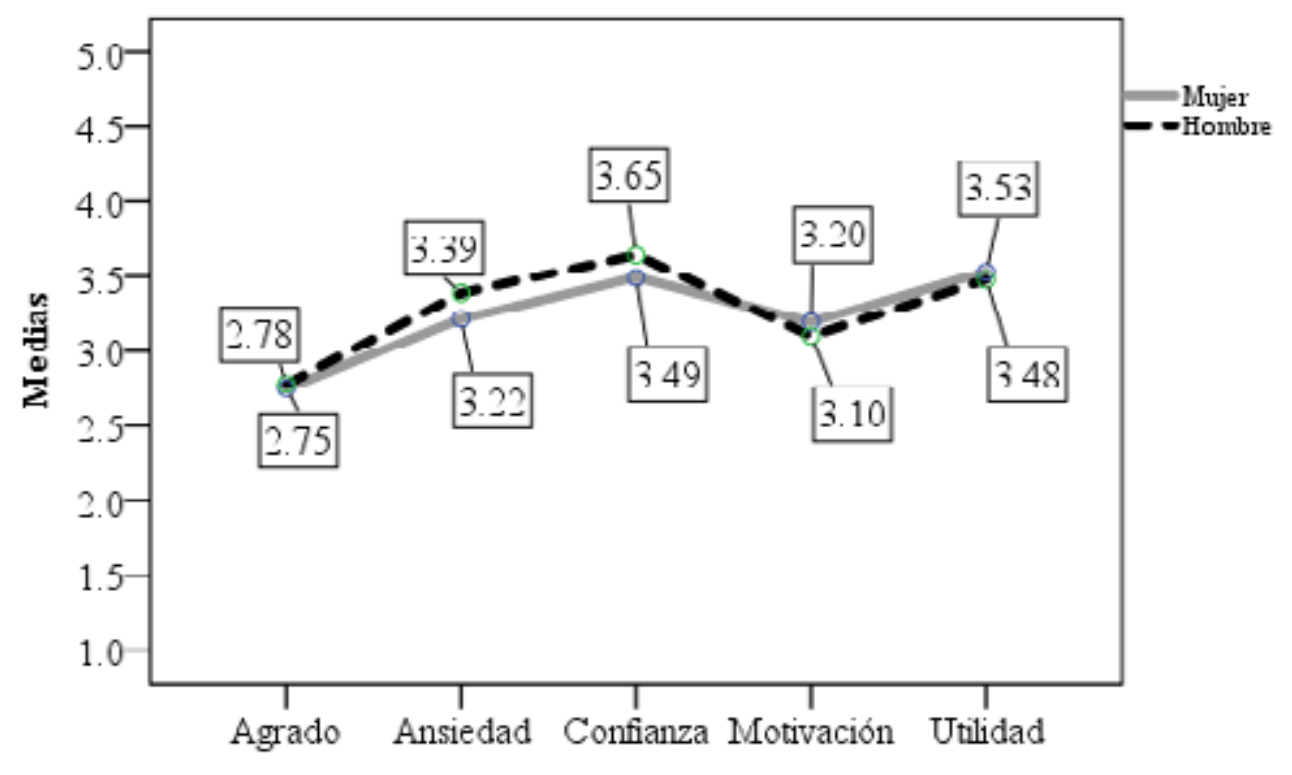

Actitud hacia la Estadística

Figura 3. Diagrama de medias de los factores de EAE-25 en mujeres y hombres.

\section{Validez de constructo concurrente}

Las correlaciones de la puntuación total y de los factores de utilidad, ansiedad y confianza con la calificación en la materia de investigación del semestre anterior fueron 
significativas, positivas y con una fuerza de asociación pequeña. Agrado y motivación fueron independientes, como se observa en la Tabla 2.

Tabla 2

Correlaciones de la EAE-25 y sus cinco factores con calificación en la materia de investigación del semestre previo y la EAI-32 y sus tres factores

\begin{tabular}{|c|c|c|c|c|c|}
\hline \multirow{2}{*}{$\begin{array}{c}\text { Actitud } \\
\text { hacia la } \\
\text { estadística }\end{array}$} & \multirow{2}{*}{$\begin{array}{l}\text { Calificación en } \\
\text { investigación }\end{array}$} & \multicolumn{4}{|c|}{ Actitud hacia la investigación } \\
\hline & & EAI-32 & AC-ID & COG & CA-II \\
\hline EAE-25 & $.128^{\mathrm{b}}(.02, .23)$ & $.524^{\mathrm{b}}(.44, .60)$ & $.439^{\mathrm{b}}(.35, .53)$ & $.372^{\mathrm{b}}(.28, .46)$ & $.486^{* * *}(.40, .57)$ \\
\hline Utilidad & $.116^{\mathrm{b}}(.02, .21)$ & $.525^{\mathrm{b}}(.43, .61)$ & $.441^{\mathrm{b}}(.33, .55)$ & $.498^{\mathrm{b}}(.42, .58)$ & $.386^{\mathrm{b}}(.27, .49)$ \\
\hline Ansiedad & $.118^{\mathrm{b}}(.01, .23)$ & $.333^{\mathrm{b}}(.24, .42)$ & $.221^{\mathrm{b}}(.12, .32)$ & $.211^{\mathrm{b}}(.11, .31)$ & $.406^{* * *}(.31, .50)$ \\
\hline Confianza & $.132^{\mathrm{b}}(.02, .24)$ & $.403^{b}(.30, .51)$ & $.343^{\mathrm{b}}(.22, .45)$ & $.346^{\mathrm{b}}(.25, .43)$ & $.319^{\mathrm{b}}(.21, .43)$ \\
\hline Agrado & $.077^{\mathrm{b}}(-.03, .19)$ & $.390^{\mathrm{b}}(.29, .48)$ & $.440^{\mathrm{b}}(.33, .53)$ & $.188^{\mathrm{b}}(.09, .28)$ & $.282^{\mathrm{b}}(.16, .39)$ \\
\hline Motivación & $.044^{\mathrm{b}}(-.06, .15)$ & $.380^{\mathrm{b}}(.27, .47)$ & $.242^{\mathrm{b}}(.14, .35)$ & $.205^{\mathrm{b}}(.09, .31)$ & $.502^{* * *}(.42, .59)$ \\
\hline
\end{tabular}

Notas: Significación contrastada bilateralmente por la prueba $t$ con 381 grados de libertad tras comprobar la normalidad bivariada por las pruebas basadas en la asimetría ( $U$-test) y curtosis ( $W$-test): ns $p>.05 * p \leq .05,{ }^{* *} p \leq .01,{ }^{* * *} p$ $\leq .001$ en un contraste a dos colas. ${ }^{b}$ Estimación por intervalo con un nivel de confianza al $95 \%$ usando el método de muestreo repetitivo de percentiles con una simulación de 2,000 muestras, al no mantenerse el supuesto de normalidad bivariada. En todos los casos el sesgo fue menor que .001. EAI-32: puntuación total de la escala de actitud hacia la investigación, AC-ID: factor afectivo-conductual de ítems directos, COG: factor cognitivo, CA-II: factor conductual-afectivo de ítems inversos.

La puntuación total en la EAE-25 presentó correlación significativa, positiva y con una fuerza de asociación alta con la EAI-32 y media con sus tres factores. Las correlaciones de los cinco factores de la EAE-25 fueron positivas y significativas con la puntuación total de EAI-32 y sus tres factores, variando de .53 a .19 con una media de .37 . Entre los factores de la EAE-25, el de utilidad tuvo las correlaciones más altas, salvo con el factor conductual afectivo de ítems inversos de la EAI-32, cuya correlación más alta fue con el factor de motivación de la EAE-25 (Tabla 2).

\section{Discusión}

El primer objetivo del estudio fue contrastar el modelo de cinco factores correlacionados propuesto por Auzmendi (1992). Su ajuste a los datos y la relación entre ajuste y parsimonia fueron buenos. Sus factores presentaron confiabilidad por consistencia interna al menos aceptable $(\omega>.70)$ y validez discriminante $(H T M T<.90)$. La validez convergente por el criterio estipulado de AVE $>.50$ se cumplió en tres de los factores (ansiedad, confianza y agrado). En utilidad, el valor de AVE quedó muy próximo a .50 y fue compensado por un valor del coeficiente omega mayor que .80 , por lo que se puede considerar que presenta validez convergente (Green y Yang, 2015). No obstante, el factor de motivación careció de validez convergente. Su varianza media extraída de .34 , que es un valor claramente menor que .50 , no fue compensado por un coeficiente omega mayor que .80 . 
¿La validez convergente de un factor de cinco ítems con una AVE de .34 podría considerarse adecuada? Fornell y Larcker (1981) fijaron el criterio de que un factor, con independencia de su número de indicadores, debe explicar más del $50 \%$ de la varianza de los mismos para considerar que posee validez convergente o certeza en que los indicadores miden una misma variable latente, es decir, que el constructo es adecuadamente medido por sus indicadores. El argumento es que la varianza atribuible al factor sea mayor que la no atribuible. Un valor de .70 en el coeficiente omega se ha estipulado como un nivel aceptable de consistencia interna o proporción de varianza verdadera contenida en las puntuaciones empíricas (Viladrich et al., 2017). No obstante, el concepto de confiabilidad por consistencia interna es muy afín al de validez convergente, especialmente estimado desde los coeficientes omega (Green y Yang, 2015) y $H$ (Domínguez, 2016). En ambos conceptos, se pretende estimar una varianza atribuible a un modelo de medida especificado con $n$ indicadores. Desde esta similitud, se podría reconsiderar el valor crítico para la AVE. Los coeficientes $\omega$ y $H$ no son invariantes con respecto al número de indicadores. Con pesos de medida estandarizados homogéneos, se obtienen valores más altos en $\omega$ y $H$ cuanto mayor es el número de indicadores. Con cinco indicadores con pesos de medida de .56 , varianza compartida con el factor de .32 , se logra que los coeficientes $\omega$ y $H$ tomen un valor justo por encima de .70, lo que implica una AVE de .32. Si se añade otro ítem con carga de .56, $\omega$ y $H$ suben a .74; con dos ítems más con cargas de .56 , ambos coeficientes suben a .77; con tres ítems más con cargas de .56 , suben a .79; y con cuatro ítems más con cargas de .56, suben a .81. El AVE no cambia con estos incrementos en ítems. Consecuentemente, una AVE mayor o igual a .32 en un factor con cinco indicadores podría considerarse como un nivel de validez convergente aceptable (Moral, 2019), como es el caso del factor de motivación.
El segundo objetivo enunciado fue describir la distribución de la escala. Como en el estudio de Flores y Auzmendi (2015), la distribución de las puntuaciones en EAE-25 se ajustó a una curva normal; así como las distribuciones de cuatro de los cinco factores. La distribución del factor de motivación mostró mayor concentración de puntuaciones en torno a la media con un histograma con más apuntamiento y un decaimiento más abrupto hacia las colas que el de una distribución normal; no obstante, esta leptocurtosis fue leve. Por tanto, la escala y sus factores pueden ser baremados por puntuaciones $T$ con media $50 \mathrm{y}$ desviación estándar de 10, al aplicarse la transformación lineal: $50+10 *$ Zx (Gaddis, Foster y Lemming, 2015). Se ha señalado que las variables cuantitativas con distribución normal son propias de fenómenos naturales y sociales sometidos a selección por presión adaptativa al entorno (Smerlak y Youssef, 2015). En este caso, se estaría hablando de un entorno académico que demanda habilidades en estadística para la compresión de los reportes de investigación y el análisis de datos.

¿Cuál es el nivel actitudinal en la muestra? Al usar criterios absolutos de interpretación, las puntuaciones en EAE-25 reflejan que aproximadamente cinco de cada diez estudiantes quedan en el intervalo de actitud neutra y la tendencia central en EAE-25 y en tres factores corresponde a un nivel neutro, lo que parece apoyar los resultados de Baños y Hurtado (2016) en España y Rodríguez Feijóo (2011) en Argentina. No obstante, la tendencia central corresponde a un nivel de aceptación en los factores de confianza y utilidad. Además, domina el polo de la aceptación con aproximadamente cuatro de cada diez estudiantes sobre el polo del rechazo con uno de cada diez, dentro de la distribución de puntuaciones en EAE-25. Por tanto, el balance final se inclina más hacia la aceptación, aunque esta polarización es menor que en los estudios de Comas et al. (2017) con estudiantes españoles de psicología y de Bautista et al. (2016) con estudiantes mexicanos de ciencias sociales. 
El tercer objetivo buscado por el estudio fue comparar los promedios entre los factores y entre ambos sexos. Por los estudios de Flores y Auzmendi (2015) y Vilá y Rubio (2016) cabría esperar un promedio más alto en ansiedad (poca ansiedad) y los más bajos en agrado, motivación y confianza. Los presentes datos no confirman estos resultados. Hubo diferencias significativas entre medias, pero las más altas fueron en utilidad y confianza y la más baja fue en falta de ansiedad. Estos alumnos mexicanos de psicología sí valoran la estadística como útil y se sienten confiados en su aplicación, pero no están exentos de ansiedad ante ella.

Con respecto al sexo, hay contradicciones entre las investigaciones, aunque finalmente domina la igualdad de medias (Estrada et al., 2004; Gil, 1999; Pérez et al., 2015; Salinas y Mayén, 2016). Los presentes datos apoyan la equivalencia del nivel actitudinal entre ambos sexos, por lo que no se requerirían baremos distintos para mujeres y hombres. Además, el promedio en la calificación en la materia de investigación en el semestre previo fue estadísticamente equivalente entre ambos sexos, lo que es consonante con los hallazgos de Stoet y Geary (2018) sobre el cierre de la brecha en la actitud y rendimiento en materias de ciencia, tecnología, ingeniería y matemáticas a medida que el nivel de desarrollo es mayor en el país. Precisamente, los datos de este estudio proceden de una de las ciudades mexicanas más desarrolladas y México se ubica en el rango 24 de las economías emergentes, de acuerdo con el reporte de crecimiento y desarrollo inclusivo del Foro Económico Mundial (World Economic Forum, 2018). En un estudio hecho en otra ciudad de México, tampoco se encontraron diferencias entre ambos sexos en el nivel de actitud global (Bautista et al., 2016).

El cuarto y último objetivo fue comprobar la validez de constructo concurrente de la EAE-25. Para este fin se consideraron dos criterios externos. Por una parte, se correlacionó con la actitud hacia la investigación y, por otra parte, con la calificación en la materia de investigación. Al ser la actitud hacia la investigación un concepto muy afín, una correlación directa y alta sería evidencia de validez de constructo convergente. La puntuación total y el factor de utilidad de la EAE-25 claramente apoyaron este criterio de validez. La fuerza de la asociación fue media en los demás factores, como también observaron Baños y Hurtado (2016).

Una correlación directa con la calificación en la materia de investigación del semestre anterior se podría tomar como evidencia de validez. Aunque la actitud es un factor facilitador, la trayectoria de estudio y la motivación hacia la carrera son mejores predictores, de ahí que la correlación puede ser baja o media (Bailey y Phillips, 2016). La escala y tres de sus cinco factores se asociaron a un mayor rendimiento en la materia de investigación del semestre anterior, con una fuerza de asociación pequeña, lo que constituye también una prueba positiva de validez de constructo concurrente. Bautista et al. (2016) hallaron una correlación más alta $(r=.32)$ en 392 estudiantes sonorenses. No obstante, el criterio fue la materia de estadística en curso que, al ser más específico y próximo temporalmente, pudo favorecer una fuerza de asociación más alta.

Como limitación del estudio, se tiene el uso de un muestreo no probabilístico, por lo que las inferencias deben tomarse con la debida cautela dentro de la población de estudiantes de psicología en la que se colectó la muestra. Su aplicación a otras poblaciones debe realizarse como hipótesis a contrastar o datos comparativos. El diseño del estudio fue transversal, por lo que no se pudo comprobar la estabilidad temporal de las puntuaciones $\mathrm{y}$ de la estructura factorial.

Como última limitación cabe señalar que la confirmación del modelo de factores correlacionados con validez discriminante entre sus cinco factores se logra al usar análisis factorial confirmatorio y la proporción HTMT, las cuales son estrategias muy adecuadas a los 
objetivos (Henseler et al., 2015) y evitan caer en errores y relaciones muy específicos a la muestra (Byrne, 2016). Cabe señalar que, si se hubiera usado el criterio de una varianza compartida entre dos factores menor que la varianza media extraída en cada factor (Fornell y Larcker, 1981), no habría validez discriminante en cinco de los diez emparejamientos; no obstante, este es un criterio menos fiable para establecer la validez divergente que una proporción hetero rasgo/mono rasgo menor o igual que .85 (Henseler et al., 2015).

Se concluye que el modelo de cinco factores correlacionados propuesto originariamente se valida entre estos estudiantes de psicología mexicanos. Las puntuaciones en la escala y los factores siguen una distribución normal, salvo el factor de motivación que muestra apuntamiento y se desvía de la normalidad. En cinco de cada diez estudiantes sus puntuaciones en EAE-25 corresponden a una actitud neutra y la actitud promedio es neutra en EAE-25 y en tres de sus cinco factores. No obstante, las puntuaciones en EAE-25 en cuatro de cada diez estudiantes corresponden a una actitud de aceptación frente a una actitud de rechazo en solo uno de cada diez. Además, los factores de confianza y utilidad muestran un promedio de aceptación, con lo que la actitud se inclina más hacia la aceptación que hacia el rechazo. El nivel actitudinal es equivalente entre mujeres y hombres. Los baremos de la escala pueden ser los mismos para ambos sexos y establecerse por medio de puntuaciones $T$ basadas en las estimaciones de la media y desviación estándar poblacionales. La escala muestra validez de constructo concurrente en relación con actitud hacia la investigación y la calificación en la materia de investigación del semestre anterior. No obstante, el potencial predictivo de la EAE-25 sobre esta última variable es muy limitado.

Se sugiere probar la estabilidad temporal del modelo factorial, comprobar la confiabilidad de la escala a través de la correlación test-retest, estimar los baremos de la escala en la población estudiada y usar este instrumento de medida en la investigación aplicada en el campo de la educación universitaria y educación media superior. Asimismo, a nivel práctico, la EAE-25 podría utilizarse como una evaluación inicial en cursos de estadística. En caso de ser necesario, podría realizarse una intervención orientada a modificar las actitudes de los estudiantes, ya que se aprende mejor aquello que produce más agrado (Baños y Hurtado, 2016). Otra variable relevante para predecir el rendimiento en estadística e investigación, y tal vez con un tamaño del efecto mayor que el de la actitud, sea la relación docente (Laudadío y Mazzitelli, 2018).

\section{Referencias bibliográficas}

Aldana, G. M., Caraballo, G. J. y Babativa, D. A. (2016). Escala para Medir Actitudes hacia la Investigación (EACIN): validación de contenido y confiabilidad. Revista Aletheia, 8(2), 104-121. https://doi. org/10.11600/21450366.8.2aletheia.104.121

American Psychological Association (2017). Ethical principles of psychologists and code of conduct. With the 2016 amendment to standard 3.04. Washington, DC: American Psychological Association Press. https://www.apa.org/ ethics/code

Auzmendi, E. (1992). Las actitudes hacia la matemática-estadística en las enseñanzas medias y universitarias. Bilbao, España: Mensajero.

Bailey, T. H. y Phillips, L. J. (2016). The influence of motivation and adaptation on students' subjective well-being, meaning in life and academic performance. Higher Education Research y Development, 35(2), 201-216. https://doi.org/10.1080/07294360.2015.1087 474

Baños, R. V. y Hurtado, M. R. (2016). Actitudes hacia la Estadística en el alumnado del grado de Pedagogía de la Universidad de Barcelona. Revista de Docencia Universitaria, 14(1), 131-150. https://doi.org/10.4995/ redu. 2016.5766

Bautista, J., Morales, M., Dórame, L. y Peralta, G. 
(2016). Un estudio sobre las actitudes hacia la estadística en estudiantes universitarios. Epistemus, 20(10), 48-54. https://doi.org/10.36790/ epistemus.v10i20.22

Bishara, A. J., y Hittner, J. B. (2015). Reducing bias and error in the correlation coefficient due to nonnormality. Educational and Psychological Measurement, 75, 785-804. https://doi. org/10.1177/0013164414557639

Byrne, B. (2016). Structural equation modeling with AMOS ( $3^{\mathrm{a}}$ ed.). New York, NY: Routledge. https://doi.org/10.4324/9781315757421

Comas, C., Martins, J. A., Nascimento, M. M. y Estrada, M. A. (2017). Estudio de las actitudes hacia la estadística en estudiantes de psicología. Bolema: Boletim de Educação Matemática, 31(57), 479-496. https://doi. org/10.1590/1980-4415v31n57a23

Domínguez, S. (2016). Evaluación de la confiabilidad del constructo mediante el Coeficiente H: Breve revisión conceptual y aplicaciones. Psychologia: Avances en la Disciplina, 10(2), 87-94. https://doi. org/10.21500/19002386.2134

Estrada, A., Batanero, C. y Fortuny, J. M. (2004). Un estudio comparado de las actitudes hacia la estadística en profesores en formación y en ejercicio. Enseñanza de las Ciencias, 22(2), 263-274.

Flores, L. W. O. y Auzmendi, E. E. (2015). Análisis de la estructura factorial de una escala de actitud hacia las matemáticas. Aula de Encuentro, 1(17), 45-77.

Fornell, C. y Larcker, D. (1981). Evaluating structural equation models with unobservable variables and measurement error. Journal of Marketing Research, 18(1), 39-50. https://doi. org/10.2307/3151312

Gaddis, B. H., Foster, J. L. y Lemming, M. R. (2015). Diversity in personality assessment norming. International Journal of Selection and Assessment, 23(1), 14-26. https://doi. org/10.1111/ijsa.12091

Gil, J. (1999). Actitudes hacia la estadística. Incidencia de las variables sexo y formación previa. Revista Española de Pedagogía, 214, 567-590.

Green, S. B. y Yang, Y. (2015). Evaluation of dimensionality in the assessment of internal consistency reliability: coefficient Alpha and Omega coefficients. Educational measurement. Issued and Practices, 34(4), 14-20. https://doi. org/10.1111/emip.12100

Greenhouse, S. W. y Geisser, S. (1959). On methods in the analysis of profile data. Psychometrika, 24, 95-112. https://doi.org/10.1007/ BF02289823

Henseler, J., Ringle, C. M. y Sarstedt, M. (2015). A new criterion for assessing discriminant validity in variance-based structural equation modeling. Journal of the Academy of Marketing Science, 43(1), 115-135. https://doi. org/10.1007/s11747-014-0403-8

Horn, J. L. (1965). A rationale and test for the number of factors in factor analysis. Psychometrika. 30, 179-185. https://doi.org/10.1007/ bf02289447

Laudadío, J. y Mazzitelli, C. (2018). Adaptación y validación del Cuestionario de Relación Docente en el Nivel Superior. Interdisciplinaria, 35(1), 153-170. https://doi.org/10.16888/ interd.2018.35.1.8

Mauchly, J. W. (1940). Significance test for sphericity of a normal n-variate distribution. The Annals of Mathematical Statistics, 11, 204-209. https://doi.org/10.1214/aoms/1177731915

Méndez, D. y Macía, F. (2007). Análisis factorial confirmatorio de la escala de actitudes hacia la estadística, Cuadernos de Neuropsicología, 3(1), 174-371.

Moral, J. (2019). Revisión de los criterios para validez convergente estimada a través de la VarianzaMediaExtraída. Psychologia, 13(2), 25-41. https://doi.org/10.21500/19002386.4119

Pérez, L. E., Aparicio, A. S., Bazán, J. L. y Abdounur, O. J. (2015). Actitudes hacia la estadística de estudiantes universitarios de Colombia. Educación Matemática, 27(3), 111-149.

Quezada, L. C., Moral, J. y Landero, R. (2019). Estudio de validación de la Escala de Actitud hacia la Investigación en estudiantes mexicanos de psicología. Evaluar, 19(1), 1-16. https://doi. org/10.35670/1667-4545.v19.n1.23874

Rodríguez Feijóo, N. (2011). Actitudes de los 
estudiantes universitarios hacia la Estadística. Interdisciplinaria, 28(2), 199-205.

Rojas, C. A., Escalera, M. E., Moreno, E. y García, A. (2017). Motivación, ansiedad, confianza, agrado y utilidad. Los factores que explican la actitud hacia las matemáticas en los estudiantes de economía. International Journal of Developmental and Educational Psychology, 2(1), 527-539. https://doi.org/10.17060/ ijodaep.2017.n1.v2.875

Ruiz de Miguel, C. (2015). Actitudes hacia la estadística de los alumnos del grado en pedagogía, educación social y maestro de educación primaria en la UCM. Educación $X X 1,18(2), 351-374$. https://doi.org/10.5944/ educXX1.12158

Salinas, J. y Mayén, S. (2016). Estudio exploratorio de las actitudes hacia la estadística en estudiantes mexicanos de bachillerato. Avances de Investigación en Educación Matemática, 10, 73-90. https://doi.org/10.35763/aiem. v0i10.130

Sidak, Z. K. (1967). Rectangular confidence regions for the means of multivariate normal distributions. Journal of the American Statistical Association, 62, 626-633. https://doi.org/ 10.1080/01621459.1967.10482935

Smerlak, M. y Youssef, A. (2017). Limiting fitness distributions in evolutionary dynamics. Journal of Theoretical Biology, 416, 68-80. https://doi. org/10.1016/j.jtbi.2017.01.005

Stoet, G. y Geary, D. C. (2018). The GenderEquality Paradox in Science, Technology, Engineering, and Mathematics Education. Psychological Science, 29, 581-593. https:// doi.org/10.1177/0956797617741719

Tejero, C. y Castro, M. (2011). Validación de la escala de actitudes hacia la estadística en estudiantes españoles de ciencias de la actividad física y del deporte. Revista Colombiana de Estadística, 34(1), 1-14.
Thabane, L., Mbuagbaw, L., Zhang, S., Samaan, Z., Marcucci, M., Ye, C., ... Goldsmith, C. H. (2013). A tutorial on sensitivity analyses in clinical trials: the what, why, when and how. BMC Medical Research Methodology, 13(92), 1-12. https://doi.org/10.1186/1471-2288-13-92

Tyler, D. E., Critchley, F., Dümbgen, L. y Oja, H. (2009). Invariant coordinate selection. Journal of the Royal Statistical Society, 71, 549-592. https://doi.org/10.1111/j.14679868.2009.00706.x

Urzúa, C. (1996). On the correct use of omnibus tests for normality. Economics Letters, 53, 247-251. https://doi.org/10.1016/S01651765(96)00923-8

Velicer, W. (1976). Determining the number of components from the matrix of partial correlations. Psychometrika, 41, 321-327. https://doi. org/10.1007/BF02293557

Vilá, R. y Rubio, M. J. (2016). Actitudes hacia la estadística en el alumnado del grado de pedagogía de la Universidad de Barcelona. Revista de Docencia Universitaria, 14(1), 131-149. https://doi.org/10.4995/redu.2016.5766

Viladrich, C., Angulo, A. y Doval, E. (2017). A journey around alpha and omega to estimate internal consistency reliability. Annals of Psychology, 33, 755-782. https://doi. org/10.6018/analesps.33.3.268401

Walker, E. R. y Brakke, K. E. (2017). Undergraduate psychology students' efficacy and attitudes across introductory and advanced statistics courses. Scholarship of Teaching and Learning in Psychology, 3(2), 132-140. http:// dx.doi.org/10.1037/st1000008

World Economic Forum (2018). The inclusive development index 2018. Summary and data highlights. Geneva, Switzerland: WEF. https:// www.weforum.org/reports/the-inclusive-development-index-2018

Recibido: 19 de mayo de 2019 Aceptado: 19 de octubre de 2020 